\title{
Long non-coding RNA XIST exerts oncogenic functions in pancreatic cancer via miR-34a-5p
}

\author{
ZHIXIA SUN ${ }^{1}$, BAOGANG ZHANG ${ }^{2}$ and TINGTING CUI ${ }^{1}$ \\ Departments of ${ }^{1}$ Ultrasound and ${ }^{2}$ Endoscopy, China-Japan Union Hospital of Jilin University, \\ Changchun, Jilin 130031, P.R. China
}

Received June 28, 2017; Accepted December 29, 2017

DOI: 10.3892/or.2018.6245

\begin{abstract}
Long non-coding RNAs (lncRNAs) have been implicated in the occurrence and progression of multiple cancers. In the present study, we investigated the role of lncRNA X inactive-specific transcript (XIST) in the development and progression of pancreatic cancer (PC). Firstly, we found that lncRNA XIST was markedly upregulated in $\mathrm{PC}$ tissues and PC cell lines, respectively. Overexpression of XIST significantly promoted the proliferation, migration and invasion, and suppressed cell apoptosis of BxPC-3 cells; knockdown of XIST significantly inhibited the proliferation, migration and invasion, and accelerated cell apoptosis of PANC-1 cells. Furthermore, BxPC-3 and PANC-1 cells transfected with different vectors were injected subcutaneously into nude mice to explore tumor formation. We found that XIST promoted tumor formation in vivo. Subsequently, we found that microRNA-34a-5p (miR-34a-5p) was downregulated in PC tissues, and predicted a poor prognosis in PC patients. In addition, the results indicated that miR-34a-5p is a target gene of XIST and was significantly negatively correlated with XIST. More importantly, we found that miR-34a-5p rescued the facilitation of malignant behavior mediated by XIST. These results indicated that XIST and miR-34a-5p may be potential effective therapeutic targets for PC.
\end{abstract}

\section{Introduction}

Pancreatic cancer (PC) is a malignant tumor with a high mortality rate and has become the leading cause of cancerrelated death (1). At present, the prognosis of patients with PC remains poor and the 5-year survival rate of $\mathrm{PC}$ patients is still low $(2,3)$. Therefore, it is urgent to understand the molecular

Correspondence to: Dr Baogang Zhang, Department of Endoscopy, China-Japan Union Hospital of Jilin University, Changchun, Jilin 130031, P.R. China

E-mail: baogangzhang0626@163.com

Key words: long non-coding RNA XIST, pancreatic cancer, miR-34a-5p, migration, invasion, proliferation mechanism of PC carcinogenesis in order to unearth reliable diagnostic and therapeutic targets for PC.

Long non-coding RNAs (lncRNAs) are non-protein-coding transcripts longer than 200 nucleotides and serve important roles in tumorigenesis $(4,5)$. In addition, mounting evidence has shown that lncRNAs are functional in every stage of tumor progression (6), and are involved in tumor growth, invasion, metastasis of multiple cancers including PC (7-9). Previous studies have shown that the lncRNAs ATB, MALAT1, HOTAIR1, H19 and HOTTIP are associated with the development and progression of PC (10-14). IncRNA $\mathrm{X}$ inactive-specific transcript (XIST) (18 kb) is important for inactivation of $\mathrm{X}$ chromosome in the development of female mammals and is a prototype of gene-silencing lncRNAs (15). Studies have indicated that lncRNA XIST is involved in the progression of glioblastoma, non-small cell lung and gastric cancer, and hepatocellular carcinoma (16-20). However, the role of IncRNA XIST in PC is not fully understood.

MicroRNAs (miRNAs) are small ( 20 nucleotides in length) non-coding RNA molecules that regulate gene expression by inhibiting translation or degrading mRNA transcripts (21). Research has shown that miRNAs participate in the occurrence and development of various diseases (22). There is a growing body of research showing that miRNAs are involved in the regulation of various biological processes including proliferation, differentiation, apoptosis, migration and invasion (23-26). Therefore, miRNAs are new and effective biomarkers for human cancer diagnostics. MicroRNA-34a-5p (miR-34a-5p) plays critical roles in the progression of various diseases. However, the biological functions and molecular mechanisms of miR-34a-5p in PC are not entirely clear.

Recently, in molecular biology, competing endogenous RNAs (abbreviated ceRNAs) regulate other RNA transcripts by competing for shared microRNAs. This has been proposed and defined as the crosstalk of RNA transcripts with miRNA response elements (MREs) $(27,28)$. An increasing number of studies have found that the interaction between IncRNAs and miRNAs occurs in many different types of cancers (29-32), adding more puzzles for miRNA and lncRNA regulatory networks. For example, lncRNA-UCA1 was found to serve as a ceRNA, promoting the cell proliferation of esophageal cancer (33); IncRNA H19 functions as a ceRNA to promote epithelial-to-mesenchymal transition of colorectal cancer (30); lncRNA HOTAIR serves as a ceRNA to regulate HER2 
expression by targeting miR-331-3p in gastric cancer (34); lncRNA FER1L4 inhibits cancer cell proliferation by functioning as a ceRNA (35).

In the present study, we investigated the expression and function of XIST in PC. We found that XIST expression was markedly upregulated in PC tissues and cells. Overexpression of XIST promoted PC cell growth, migration, invasion and metastasis; knockdown of XIST suppressed PC cell growth, migration, invasion and metastasis, implying a possible role of lncRNA XIST as an oncogene in PC. Moreover, we demonstrated that XIST was involved in the proliferation, migration, invasion and progression of PC by targeting miR-34a-5p.

\section{Materials and methods}

Ethics statement and specimens. Paired PC $(\mathrm{n}=139)$ and corresponding non-tumor control tissues were collected from the Japan Union Hospital of Jilin University from 2013 to 2015. The present study was approved by the Ethics Committee of Japan Union Hospital of Jilin University, and written informed consent was obtained from all patients. All tissue samples were immediately conserved at $-80^{\circ} \mathrm{C}$ after washing with sterile phosphate-buffered saline (PBS).

Cell culture. Six PC cell lines (PANC-1, ASPC-1, MIA PaCa-2, HPAC, CFAPC-1 and BxPC-3), and human pancreatic ductal epithelial (HPDE), and 293T cells were purchased from the Institute of Biochemistry and Cell Biology of the Chinese Academy of Sciences (Shanghai, China). Cells were grown in Dulbecco's modified Eagle's medium (DMEM) supplemented with 10\% fetal bovine serum (FBS) (both from Invitrogen Life Technologies, Carlsbad, CA, USA) and $100 \mathrm{U} / \mathrm{ml}$ penicillin and $100 \mu \mathrm{g} / \mathrm{ml}$ streptomycin in an atmosphere of $5 \% \mathrm{CO}_{2}$ at $37^{\circ} \mathrm{C}$.

Lentiviral vector construction. Human XIST full-length DNAs were amplified by PCR from mRNA of BxPC-3 cells. Then, the cDNAs were inserted into a pcDNA3.1 vector. Enhanced green fluorescent protein (EGFP) served as a control. Specific shRNA against XIST and luciferase shRNA (Luc-shRNA; control) were synthesized and validated effective by RiboBio Co. (Guangzhou, China). The VSV-G pseudotyped lentiviruses were produced by co-transfecting $293 \mathrm{~T}$ cells with lentivirus expression plasmid and packaging plasmids (pMD2.G, pMDL-G/P-RRE and pRSV-REV). Cells $\left(5 \times 10^{4}\right)$ were then transduced with the lentiviruses in the presence of $8 \mu \mathrm{g} / \mathrm{ml}$ Polybrene (Sigma-Aldrich, St. Louis, MO, USA).

Cell transfection. For plasmid transfection, BxPC-3 cells $\left(2 \times 10^{5}\right.$ cells/well) were seeded in 6-well plates, and transfected with XIST or the control using Lipofectamine ${ }^{\mathrm{TM}} 3000$ (Invitrogen, Carlsbad, CA, USA) respectively according to the manufacturer's protocols. Likewise, PANC-1 cells were transfected with shXIST or control, respectively.

For miRNA transfection, miR-34a-5p mimics, inhibitor and negative control (miR-34a-5p NC) were purchased from GenePharma Co., Ltd. (Shanghai, China). Cells were transfected with miR-34a-5p mimics, miR-34a-5p inhibitors and miR-34a-5p NC respectively using Lipofectamine ${ }^{\mathrm{TM}} 3000$, respectively, according to the manufacturer's protocols.
Table I. Primer sequences for qRT-PCR analysis.

\begin{tabular}{|c|c|}
\hline Gene name & Primer sequences \\
\hline $\operatorname{miR}-34 a-5 p$ & $\begin{array}{l}\text { Forward 5'-GGGGTGGCAGTGTCTTAGC-3' } \\
\text { Reverse 5'-CAGTGCGTGTCGTGGAGT-3' }\end{array}$ \\
\hline U6 & $\begin{array}{l}\text { Forward 5'-CTCGCTTCGGCAGCACA-3' } \\
\text { Reverse 5'-AACGCTTCACGAATTTGCGT-3' }\end{array}$ \\
\hline XIST & $\begin{array}{l}\text { Forward 5'-AGCTCCTCGGACAGCTGTAA-3 } \\
\text { Reverse 5'-CTCCAGATAGCTGGCAACC-3' }\end{array}$ \\
\hline GAPDH & $\begin{array}{l}\text { Forward 5'-TGTTCGTCATGGGTGTGAAC-3' } \\
\text { Reverse 5'-ATGGCATGGACTGTGGTCAT-3' }\end{array}$ \\
\hline
\end{tabular}

miR-34a-5p, microRNA-34a-5p; XIST, X inactive-specific transcript.

RNA isolation and quantitative real-time PCR ( $q R T-P C R)$. Total RNA from PC tissues or cells was isolated using TRIzol reagent (Invitrogen) after treatments. Then, reverse transcription reaction was performed with Revert Aid First Strand cDNA Synthesis kit (Thermo Fisher Scientific Inc., Rockford, IL, USA) and random primers to provide cDNA products. The thermocycling conditions were $25^{\circ} \mathrm{C}$ for $5 \mathrm{~min}$; $42^{\circ} \mathrm{C}$ for $60 \mathrm{~min} ; 70^{\circ} \mathrm{C}$ for $10 \mathrm{~min}$ for the reverse transcription. qRT-PCR assays were performed using SYBR Premix Ex Taq (Takara, Tokyo, Japan), primers and cDNA templates on the Applied Biosystems 7500 Real-Time PCR system [Applied Biosystems Inc. (ABI) Carlsbad, CA, USA). Each individual sample was performed in triplicate and the expression levels were quantified using the comparative cycle threshold (CT) method. Results were normalized to GAPDH expression and RNA enrichments were calculated using the equation $2^{-\Delta \Delta C t}(36)$. Specific primers for XIST and miR-34a-5p were designed and synthesized by RiboBio Co. The primer sequences used in the present study are shown in Table I.

Luciferase reporter assay. $293 \mathrm{~T}$ cells $\left(1 \times 10^{5}\right.$ cells/well) were placed in a 24-well plate, and co-transfected $200 \mathrm{ng}$ of either pGL3-XIST-wt or pGL3-XIST-Mut vector and $80 \mathrm{ng}$ of either miR-34a-5p or miR-NC. After $48 \mathrm{~h}$ for transfection, cells were harvested and luciferase activities were measured with the Dual-Luciferase Reporter Assay System (Promega, Wisconsin, WI, USA). All transfection experiments were conducted in triplicate.

Cell viability. Cell viability was assessed by 3-(4,5-dimethylthiazol-2-yl)-2,5-diphenyl-tetrazolium bromide (MTT) assay. The treated cells $(4,000$ cells/well) were seeded in a $96-$ well plate and cultured in complete medium for 1,2,3,4 and 5 days. MTT $(0.5 \mathrm{mg} / \mathrm{ml})$ was added to each well and incubation was carried out at $37^{\circ} \mathrm{C}$ for $4 \mathrm{~h}$. Afterward, the supernatant was carefully aspirated and $100 \mu 1$ of dimethyl sulfoxide (DMSO) was added. The absorbance was measured at $490 \mathrm{~nm}$ using a microplate reader. All experiments were repeated $\geq 3$ times.

Colony formation assay. For the colony formation assay, the treated BxPC-3 and PANC-1 cells were seeded in 6-well plates at a density of 500 cells/well after transfection with different 
vectors. After 14 days, the cells were fixed with a $4 \%$ paraformaldehyde solution and stained with crystal violet. The total number of colonies in each plate from three independent transfections was counted under an inverted microscope to evaluate the colony formation ability.

Wound-healing assay. BxPC-3 or PANC-1 cells were transfected with different vectors and seeded in 6-well plates. Small linear wounds were created by removing a line of cells with a disinfected Eppendorf tip. After removing cell debris by washing with FBS-free medium, the wound areas were photographed under a microscope. Three different positions of distance between the two edges of the wound were calculated and analyzed by image analysis software (National Institute of Health, Bethesda, MD, USA).

Migration and invasion assays. Cell migration and invasion assays were performed using the Transwell assay according to the manufacturer's instructions. BxPC-3 or PANC-1 cells $\left(5 \times 10^{4}\right.$ cells/well) were transfected with different vectors and seeded in the upper compartment of the Transwell and incubated in serum-free media, and the lower compartment was filled with complete medium supplemented with $10 \%$ FBS. After $48 \mathrm{~h}$ of incubation at $37^{\circ} \mathrm{C}$, non-invading cells were removed. The migratory and invasive cells on the bottom surface of the filters were fixed using $4 \%$ paraformaldehyde, and stained with $0.1 \%$ crystal violet solution. Four randomly selected fields of the fixed cells were counted for each group. The experiments were performed in triplicate.

Flow cytometric analysis. Treated cells were washed with 1X PBS, trypsinized and fixed with $70 \%$ ethanol for $30 \mathrm{~min}$ on ice. RNA was degraded with $20 \mathrm{mg} / \mathrm{ml}$ RNase (Sigma-Aldrich) for $1 \mathrm{~h}$ at $37^{\circ} \mathrm{C}$. DNA was then labeled with $20 \mathrm{mg} / \mathrm{ml}$ propidium iodide (PI; Sigma-Aldrich). The cell cycle images were obtained and analyzed using FACSCalibur (BD Biosciences, Franklin Lakes, NJ, USA) and FlowJo software (Tree Star, Inc., Ashland, OR, USA).

Tumor formation assay in nude mice. The BxPC-3 cells were transfected with control plasmid or XIST plasmid. The PANC-1 cells were infected with control or shXIST plasmid, respectively. Four-week-old male nude mice were purchased from the National Laboratory Animal Center (Shanghai, China) and divided into four groups for subcutaneous injection using BxPC-3 or PANC-1 cells. Animals were sacrificed 40 days after injection and tumors were collected for measurement of the volume every 10 days. The tumor volume (V) was calculated by the formula: $\mathrm{V}\left(\mathrm{mm}^{3}\right)=$ length $\mathrm{x}$ width ${ }^{2} / 2$. All experiments were performed strictly in accordance with a protocol approved by the Administrative Panel on Laboratory Animal Care of Jilin University.

Statistical analysis. The data were analyzed by the Student's t-test and one-way analysis of variance (ANOVA) using SPSS 15.0 software (SPSS, Inc., Chicago, IL, USA). Pearson's correlation coefficient was used to calculate the correlation between miR-34a-5p and XIST. The Kaplan-Meier method test was utilized for survival analysis. Each experiment was repeated at least three times. All results were summarized and
Table II. Relationship between IncRNA-XIST expression level $(\Delta \mathrm{Ct})$ and clinicopathological characteristics of the PC patients.

\begin{tabular}{lccc}
\hline & & \multicolumn{1}{l}{ lncRNA-XIST } & \\
Characteristics & $\mathrm{n}(\%)$ & Mean \pm SD & P-value \\
\hline Total no. of patients & 139 & & \\
Age (years) & & & 0.101 \\
$>60$ & $59(42.4)$ & $11.85 \pm 1.73$ & \\
S60 & $80(57.6)$ & $11.19 \pm 2.69$ & \\
Sex & & & 0.232 \\
Male & $89(64.0)$ & $11.59 \pm 1.75$ & \\
Female & $50(36.0)$ & $12.02 \pm 2.45$ & \\
Lymphatic metastasis & & & 0.235 \\
N0 & $94(67.6)$ & $12.05 \pm 1.49$ & \\
N1-N2 & $45(32.4)$ & $11.71 \pm 1.73$ & \\
Distal metastasis & & & $\mathbf{0 . 0 0 3}^{\mathrm{b}}$ \\
M0 & $105(75.5)$ & $11.98 \pm 1.06$ & \\
M1 & $34(24.5)$ & $11.14 \pm 2.16$ & \\
TNM stage & & & $\mathbf{0 . 0 1 2}^{\mathrm{a}}$ \\
0, I, II & $91(65.5)$ & $11.72 \pm 1.58$ & \\
III, IV & $48(34.5)$ & $10.96 \pm 1.83$ & \\
\hline
\end{tabular}

${ }^{\mathrm{a}} \mathrm{P}<0.05,{ }^{\mathrm{b}} \mathrm{P}<0.01$. PC, pancreatic cancer; lncRNA, long non-coding RNA; $\mathrm{XIST}, \mathrm{X}$ inactive-specific transcript; TNM, tumor-node-metastasis.

are presented as means $\pm \mathrm{SD}$. $\mathrm{P}<0.05$ was considered statistically significant.

\section{Results}

Upregulation of IncRNA-XIST predicts a poor prognosis in PC patients. In the present study, we determined lncRNA-XIST expression in 139 PC patients and analyzed the relationship between XIST expression and clinicopathological characteristics [age, sex, lymphatic metastasis, distal metastasis and TNM stage] of the PC patients. The results showed that lncRNA-XIST was significantly upregulated in tumor tissues compared with that noted in the matched adjacent normal tissues $(\mathrm{P}<0.001$; Fig. 1A). We also found that lncRNA-XIST expression was significantly related to the tumor-nodemetastasis (TNM) stage, and was higher in the TNM I, II, III and IV stages than that noted in the normal group $(\mathrm{P}<0.05$, $\mathrm{P}<0.01, \mathrm{P}<0.001$; Fig. 1B). Therefore, our results indicated that lncRNA-XIST expression is correlated with the malignant degree of PC. The Chi-square analysis indicated that the expression level of IncRNA-XIST was positively correlated with TNM stage $(\mathrm{P}<0.05)$ and distal metastasis $(\mathrm{P}<0.01)$, suggesting that lncRNA-XIST may be a potential biomarker for PC (Table II). In addition, PC patients with a high expression of XIST had a shorter overall survival than patients with low XIST expression ( $\mathrm{P}<0.001$; Fig. 1C). The results also indicated that IncRNA-XIST expression was significantly downregulated in 67 post-operation patients compared with pre-operation patients $(\mathrm{P}<0.001$; Fig. 1D). All the above results 

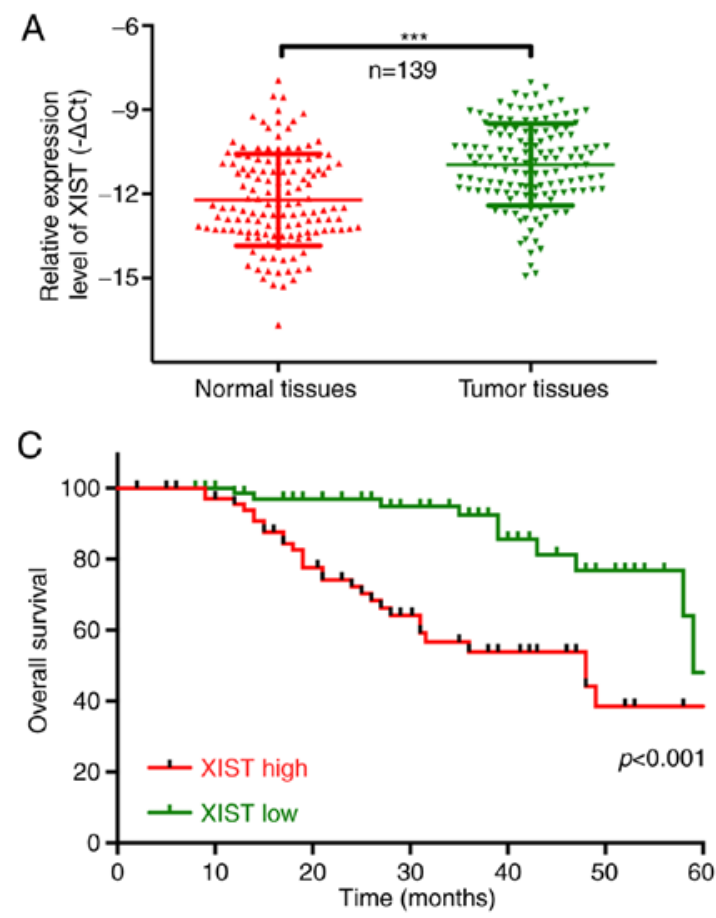
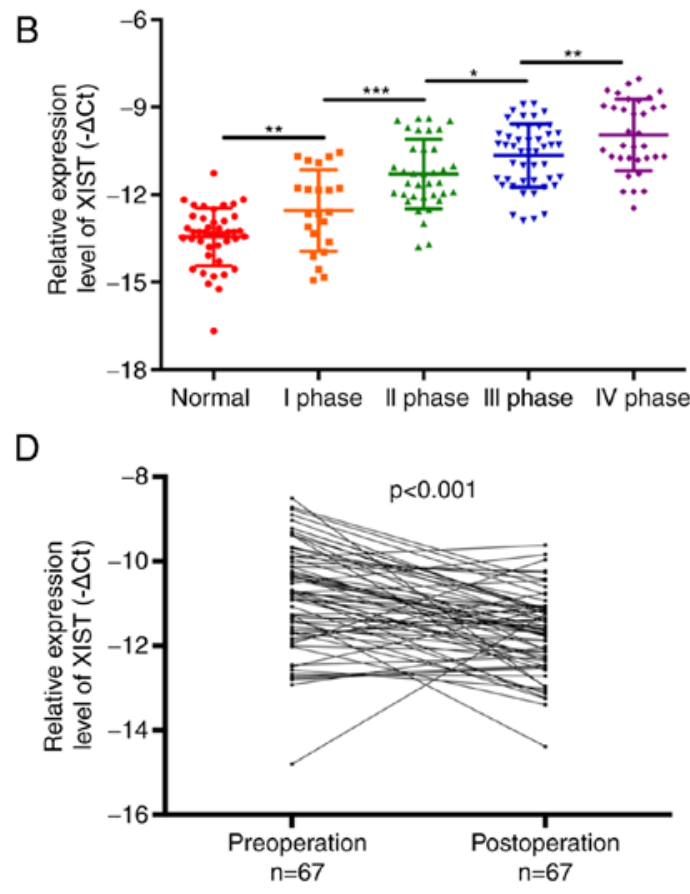

Figure 1. Upregulation of IncRNA-XIST predicts the poor prognosis of PC patients. (A) The expression level of lncRNA-XIST was detected using qRTPCR assay in PC tissues and their corresponding adjacent samples $\left(\mathrm{n}=139,{ }^{* * *} \mathrm{P}<0.001\right)$. (B) lncRNA-XIST expression was determined by qRT-PCR assay in tissues with different tumor-node-metastasis (TNM) stages $\left({ }^{*} \mathrm{P}<0.05,{ }^{* * *} \mathrm{P}<0.01,{ }^{* * *} \mathrm{P}<0.001\right)$. (C) Patients with high lncRNA-XIST expression had a worse prognosis when compared with patients with low lncRNA-XIST expression $(\mathrm{P}<0.001)$. (D) Expression of IncRNA-XIST was analyzed using qRT-PCR assay in 67 patients with PC pre-operation and post-operation ( $\mathrm{P}<0.001)$. PC, pancreatic cancer; IncRNA, long non-coding RNA; XIST, X inactive-specific transcript.

demonstrated that a high expression level of XIST is associated with poor prognosis.

XIST promotes PC tumor cell growth in vitro. To further explore the oncogenic roles of XIST on PC in vitro, the expression level of IncRNA-XIST was detected by qRT-PCR in human pancreatic ductal epithelial (HPDE) cells and PC cell lines (PANC-1, ASPC-1, MIA PaCa-2, HPAC, CFAPC-1, and BxPC-3). The results revealed that IncRNA-XIST expression was increased in the $\mathrm{PC}$ cell lines compared with that noted in the HPDE cells $(\mathrm{P}<0.05, \mathrm{P}<0.01, \mathrm{P}<0.001$; Fig. 2A). According to the lncRNA-XIST expression level in PC cells, we established PC cell lines (BxPC-3 and PANC-1) with XIST stable overexpression or knockdown (shXIST), respectively. The results indicated that lncRNA-XIST may play a critical role in the progression of PC. As shown in Fig. 2B, the expression level of XIST was increased in the BxPC-3 cells transfected with XIST-overexpressing plasmid compared with the control. XIST expression was decreased in PANC-1 cells transfected with shXIST compared with the control. Overexpression of XIST significantly promoted BxPC-3 cell viability, and knockdown of XIST significantly inhibited PANC-1 cell viability $(\mathrm{P}<0.01, \mathrm{P}<0.001$; Fig. $2 \mathrm{C}$ and $\mathrm{D})$. Then, we evaluated the effect of XIST on cell cycle distribution of the PC cells. In agreement with the above results, we found that overexpression of XIST observably decreased the percentage of cells in the G0/G1 stage and increased the percentage of cells in the S and G2/M stages; knockdown of XIST significantly induced cell cycle arrest in the G0/G1 stage and decreased the percentage of cells in the $\mathrm{S}$ and $\mathrm{G} 2 / \mathrm{M}$ stages $(\mathrm{P}<0.05, \mathrm{P}<0.01$; Fig. $2 \mathrm{E})$.
XIST accelerates the migration and invasion abilities of $P C$. We further studied whether XIST functions as an oncogene. Wound healing assays found that overexpression of XIST significantly promoted the wound healing ability and knockdown of XIST significantly decreased this ability $(\mathrm{P}<0.001$; Fig. 3A). Moreover, we used Transwell assay to explore the effects of XIST on the migration and invasion of BxPC-3 and PANC-1 cells. The results indicated that XIST obviously promoted the migration and invasion abilities of the BxPC-3 cells; knockdown of XIST markedly inhibited the migration and invasion abilities of the PANC-1 cells $(\mathrm{P}<0.01, \mathrm{P}<0.001$; Fig. 3B and C).

XIST promotes tumor formation in vivo. To further assess the growth effect of XIST on PC, we examined the tumorigenicity in nude mice. The result showed that overexpression of XIST in BxPC-3 cells accelerated tumor growth in the nude mouse model, and knockdown of XIST in PANC-1 cells inhibited tumor growth in the nude mouse model $(\mathrm{P}<0.01, \mathrm{P}<0.001$; Fig. 4). At 10, 15, 20, 25, 30, 35 and 40 days after injection, the tumors were removed. The tumor volume was accordance with above observation. From the above results, we suggest that XIST is an oncogene and promotes tumor formation.

Downregulation of $\mathrm{miR}-34 a-5 p$ predicts a poor prognosis in $P C$ patients. Similarly, we found that miR-34a-5p was significantly decreased in tumor tissues compared with that noted in the matched adjacent normal tissues $(\mathrm{P}<0.001$; Fig. $5 \mathrm{~A})$. The results also showed that miR-34a-5p expression was significantly related to TNM stage, and was lower in the TNM I, II, III 

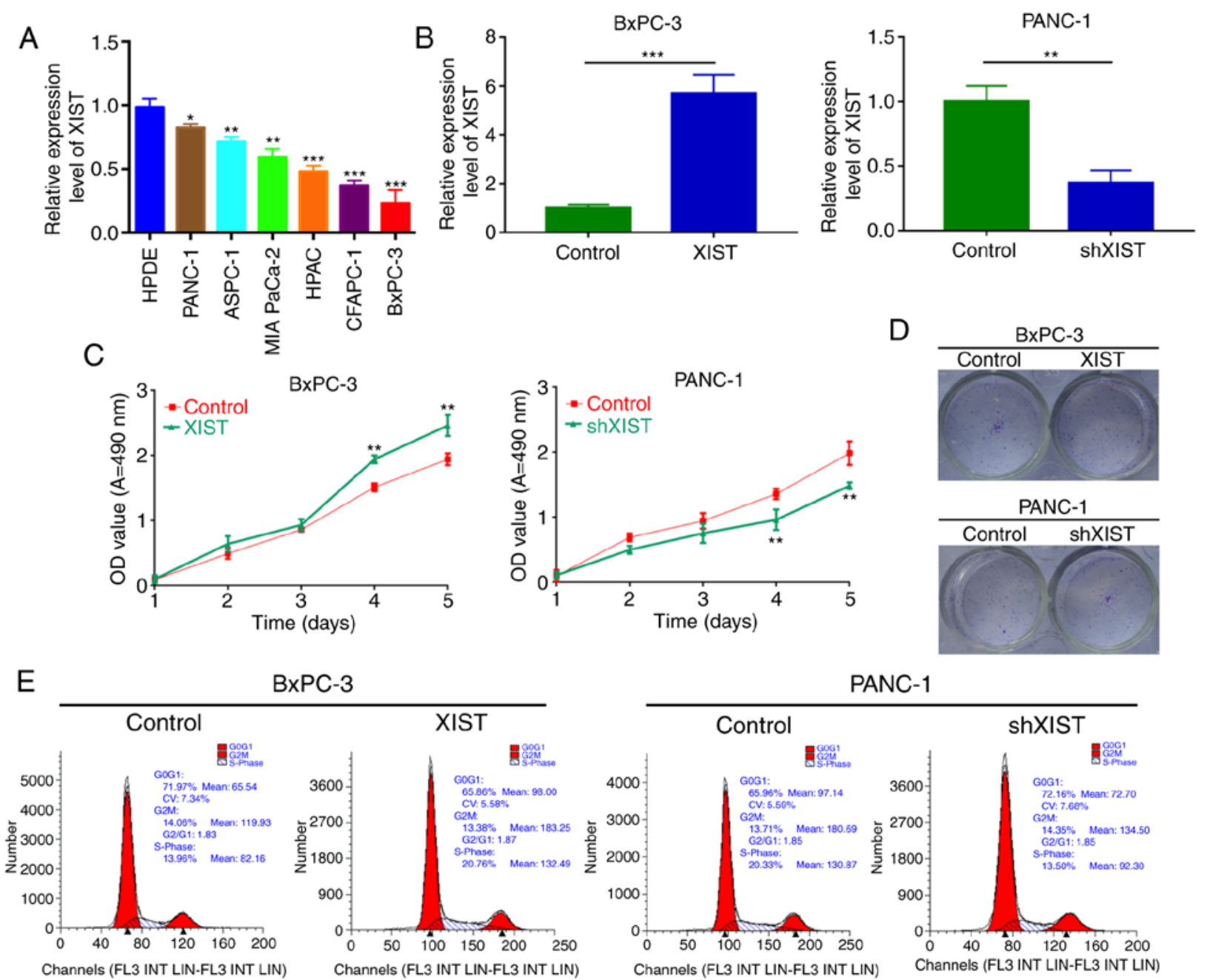

BxPC-3

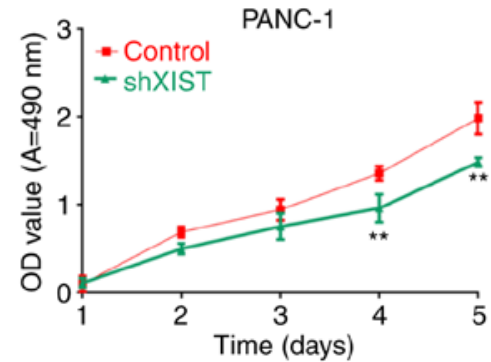

$\mathrm{D}$
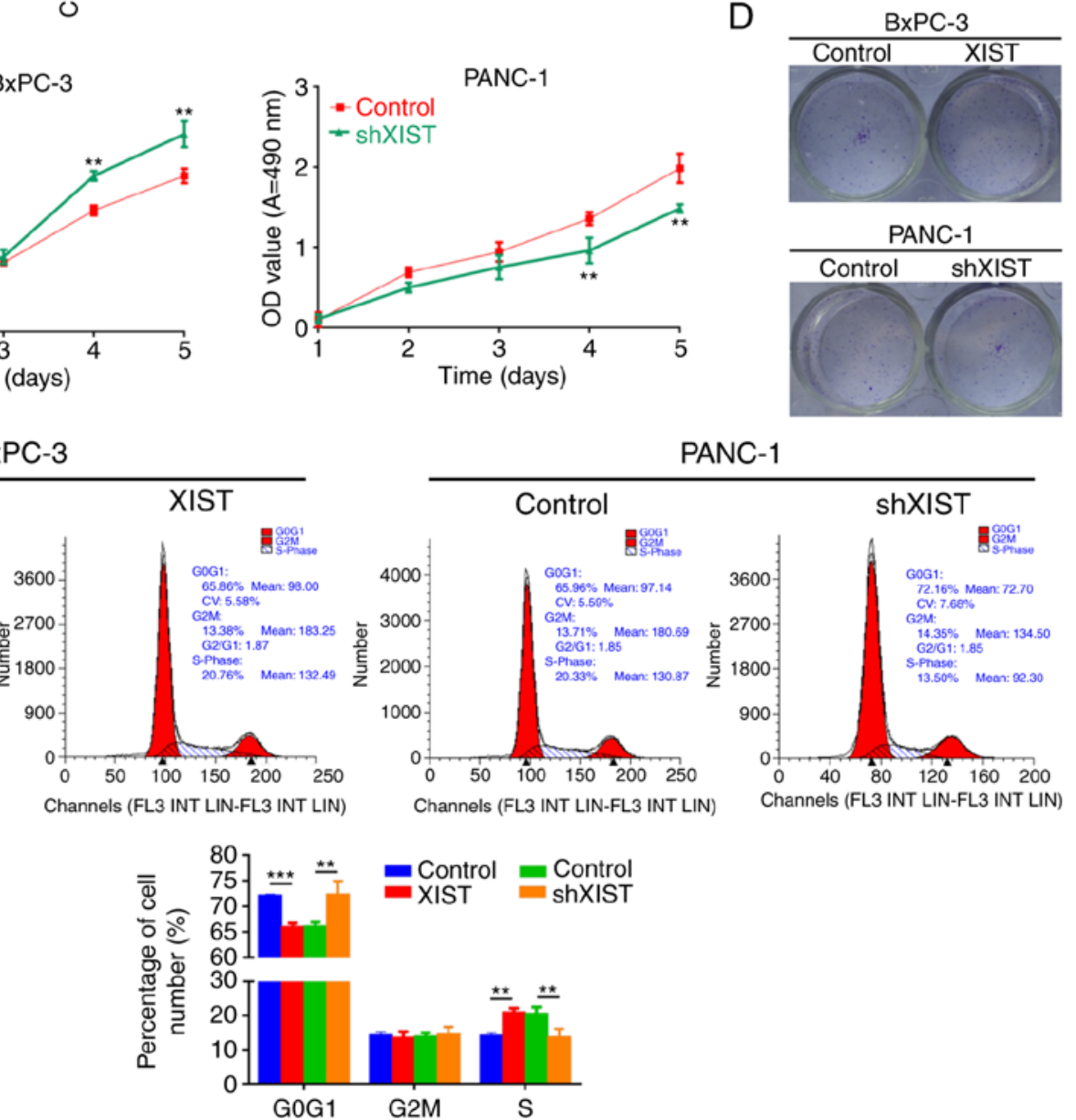

Figure 2. XIST promotes PC cell growth in vitro. (A) Expression level of lncRNA-XIST was detected by qRT-PCR assay in human pancreatic ductal epithelial (HPDE) cells and PC cell lines (PANC-1, ASPC-1, MIA PaCa-2, HPAC, CFAPC-1 and BxPC-3). Each assay was performed for at least three biological replicates ( $\left.{ }^{*} \mathrm{P}<0.05,{ }^{* *} \mathrm{P}<0.01,{ }^{* * * *} \mathrm{P}<0.001\right)$. (B) XIST expression was examined by qRT-PCR assay in BxPC-3 cells transfected with XIST plasmid and control, and PANC-1 cells transfected with shXIST and control. Relative expression was normalized to GAPDH expression ("P<0.01). (C) MTT assay was performed to measure the viability of BxPC-3 and PANC-1 cells treated as in $\mathrm{B}\left({ }^{* *} \mathrm{P}<0.01\right)$. (D) Colony formation assay was used to detect the proliferation ability of the transfected cells. (E) Cell Cycle distribution was analyzed by flow cytometry in BxPC-3 and PANC-1 cells after transfection $\left({ }^{* *} \mathrm{P}<0.01,{ }^{* * * *} \mathrm{P}<0.001\right) . \mathrm{PC}$, pancreatic cancer; IncRNA, long non-coding RNA; XIST, X inactive-specific transcript.

and IV stage than that in the normal group $(\mathrm{P}<0.05, \mathrm{P}<0.01$, $\mathrm{P}<0.001$, Fig. 5B). The Chi-square analysis also indicated that miR-34a-5p expression was related to lymphatic metastasis $(\mathrm{P}=0.006)$ and TNM stage $(\mathrm{P}=0.009)$, suggesting that miR-34a-5p may be a potential biomarker for PC (Table III). Furthermore, high-expression of miR-34a-5p in patients with $\mathrm{PC}$ had a longer overall survival than patients with low miR34a-5p expression $(\mathrm{P}<0.01$; Fig. $5 \mathrm{C})$. miR-34a-5p expression was significantly increased in 67 post-operation patients compared with that noted in pre-operation patients $(\mathrm{P}<0.001$; Fig. 5D).
miR-34a-5p abrogates the facilitation of malignant behavior mediated by XIST. Furthermore, Pearson's correlation coefficient indicated that miR-34a-5p was significantly negatively correlated with XIST $\left(\mathrm{R}^{2}=0.2175, \mathrm{P}<0.001\right.$; Fig. 6A). We hypothesized that miR-34a-5p may play roles in organisms by targeting XIST. 293T cells were co-transfected with luciferase construct (pGL3-Control, pGL3-XIST WT or pGL3-XIST-Mut) and negative control (miR-control) or miR-34a-5p mimics, respectively. Luciferase reporter assay was performed to detect the regulatory relationship between miR-34a-5p and XIST. The results showed that miR-34a-5p 
A

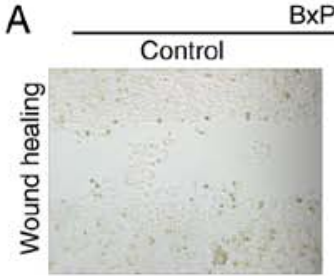

B

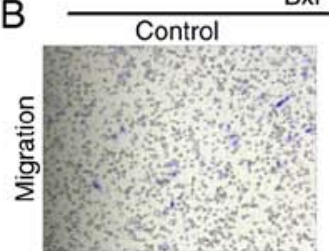

BxPC-3

BxPC-3

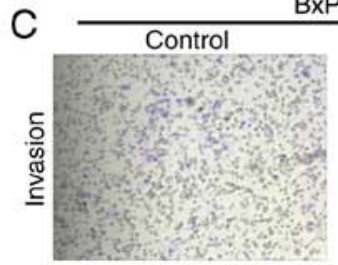

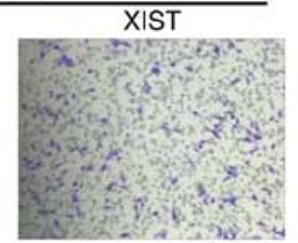

XIST

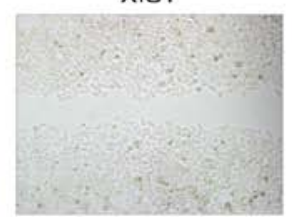

XIST

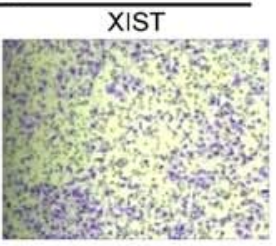

PANC-1

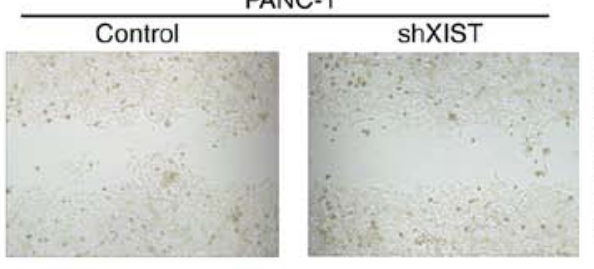

PANC-1

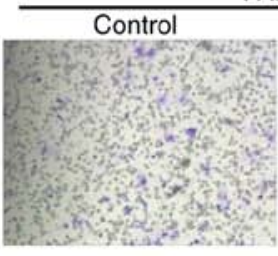

PANC-1

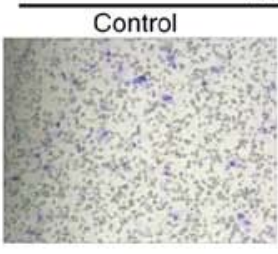

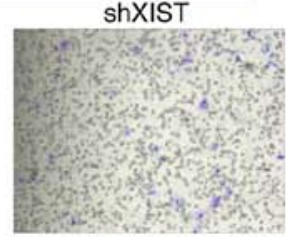
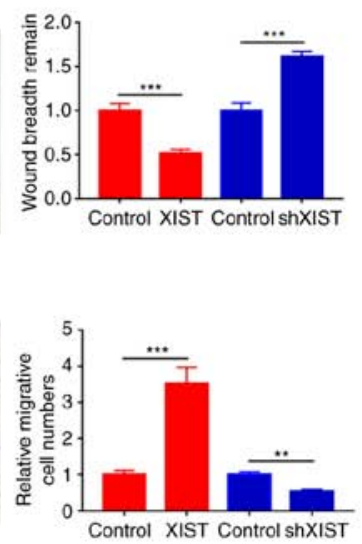

Control XIST Control shXIST
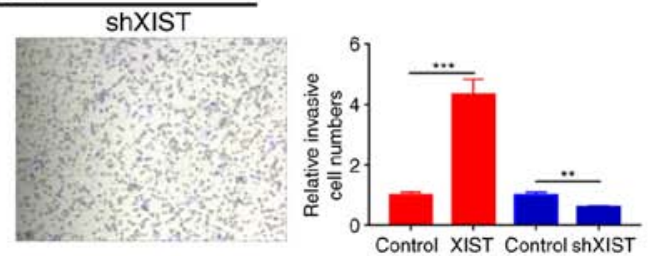

Figure 3. XIST accelerates the migration and invasion abilities of PC. BxPC-3 cells were transfected with XIST or control, and PANC-1 cells were transfected with shXIST or control, respectively. (A) Wound healing assay was used to detect the migration capability $\left({ }^{* * *} \mathrm{P}<0.001\right)$. (B and C) Transwell assays were performed to detect the migration and invasion abilities ${ }^{* *} \mathrm{P}<0.01,{ }^{* * * *} \mathrm{P}<0.001$ compared with the control group). PC, pancreatic cancer; XIST, $\mathrm{X}$ inactivespecific transcript;

A
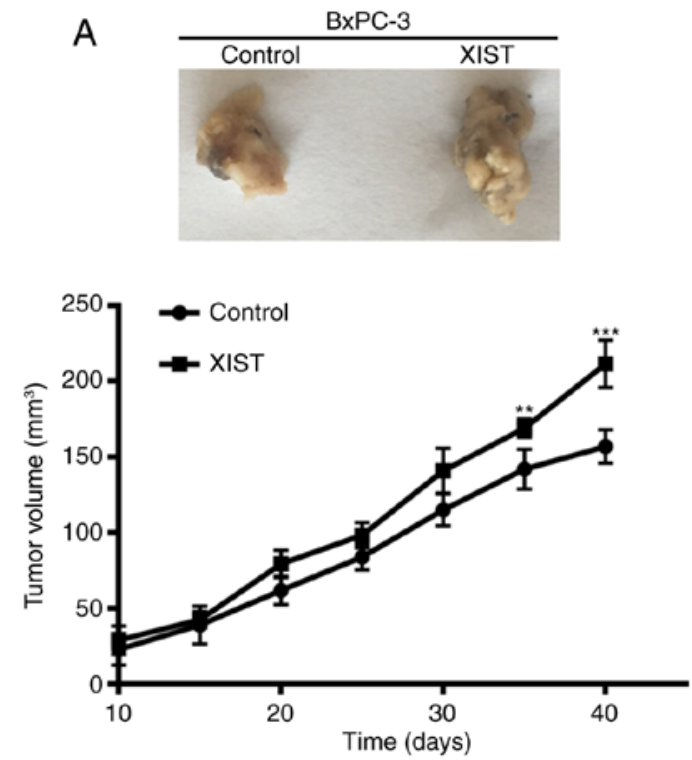

B
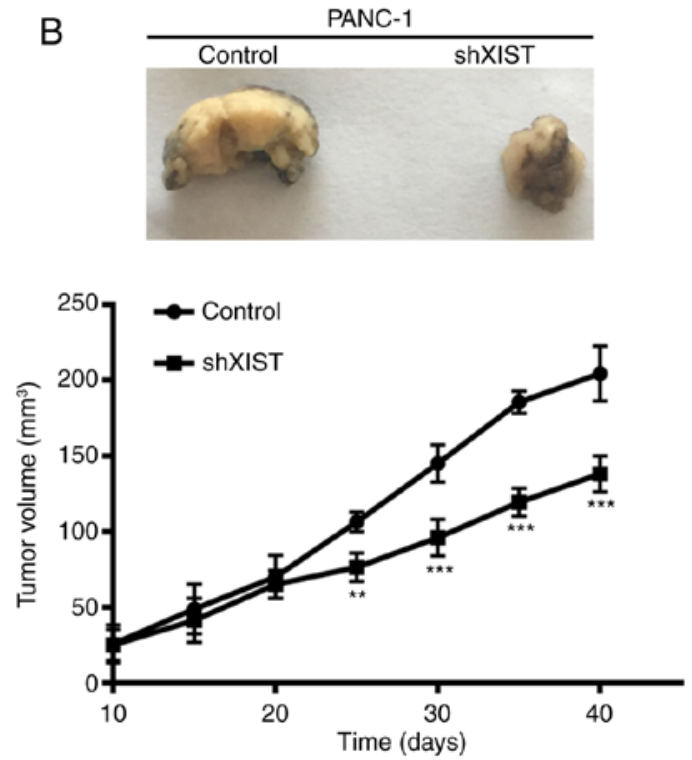

Figure 4. XIST promotes tumor formation in vivo. BxPC-3 cells were transfected with XIST or control, and PANC-1 cells were transfected with shXIST or control, respectively. The transfected cells were inoculated into the nude mouse for 40 days. Finally, the nude mice were sacrificed and the tumors excised. Dissected tumors were photographed every 10 days. The tumor volume was also measured $\left({ }^{* *} \mathrm{P}<0.01,{ }^{* * *} \mathrm{P}<0.001\right.$ compared with the control group). XIST, $\mathrm{X}$ inactive-specific transcript.

markedly decreased the relative fluorescence value in Luc-wt reporter constructs, suggesting that miR-34a-5p was a target gene of XIST ( $\mathrm{P}<0.001$; Fig. 6B). In addition, we found that upregulation of XIST expression significantly decreased miR-34a-5p expression in BxPC-3 cells, and miR-34a-5p mimics significantly increased miR-34a-5p expression inhibited by XIST. Meanwhile, knockdown of XIST significantly increased miR-34a-5p expression in PANC-1 cells, and miR-34a-5p inhibitors significantly decreased miR-34a-5p expression induced by XIST $(\mathrm{P}<0.01, \mathrm{P}<0.001$; Fig. 6C). Furthermore, we used Transwell chamber inserts to explore the effects of miR-34a-5p and XIST on the migration and invasion of BxPC-3 and PANC-1 cells. The results indicated that overexpression of XIST promoted cell proliferation, while miR-34a-5p mimics inhibited cell proliferation induced by XIST; knockdown of XIST decreased cell proliferation, and 

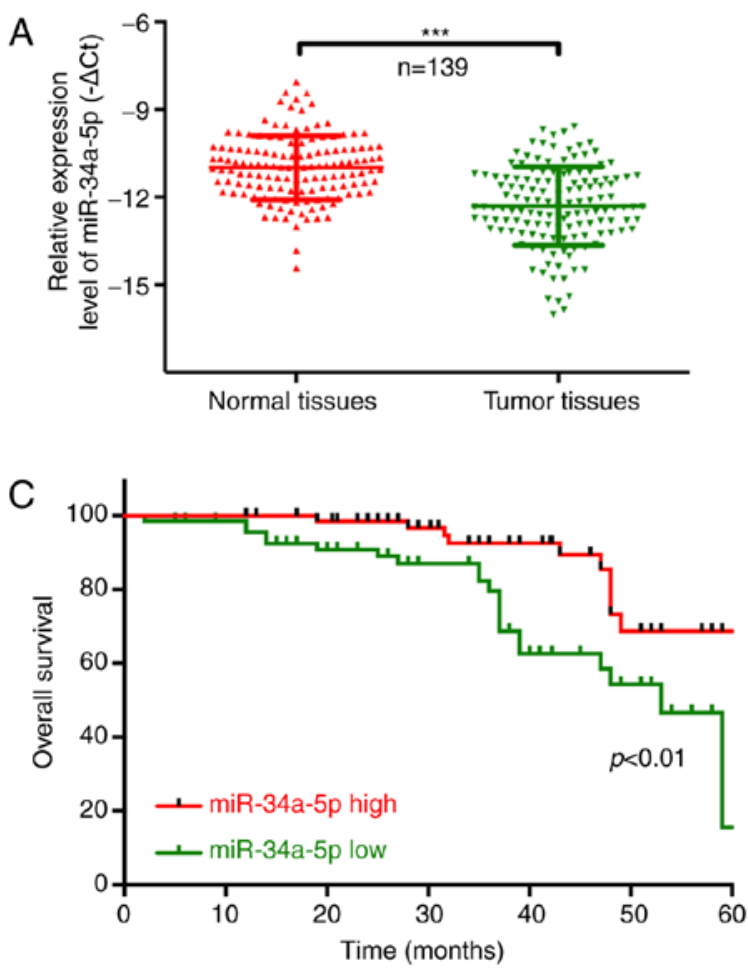
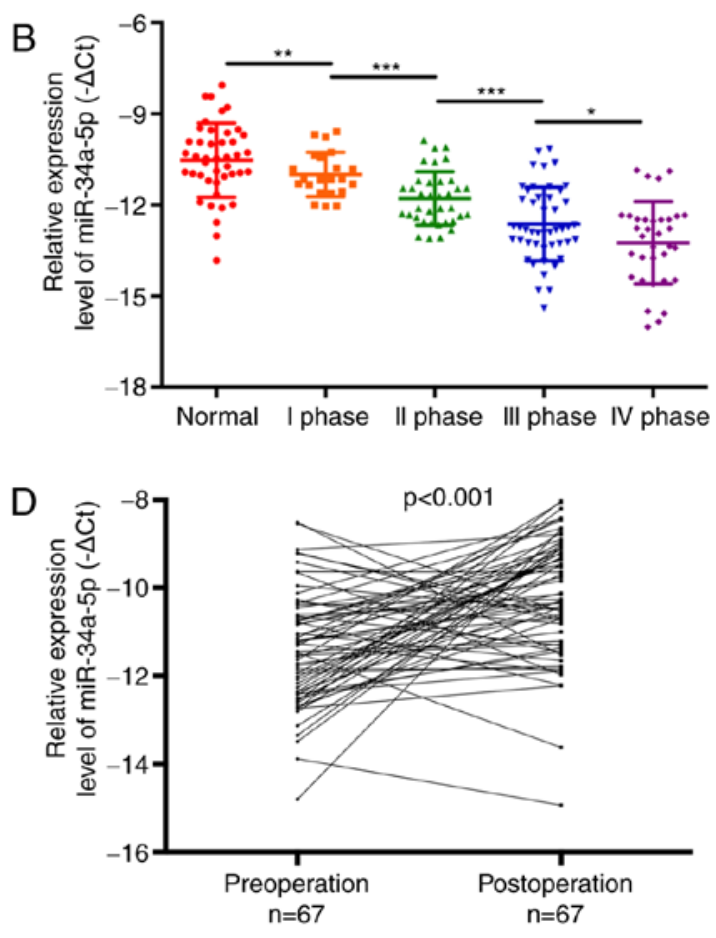

Figure 5. Downregulation of miR-34a-5p predicts a poor prognosis in PC patients. (A) The expression level of miR-34a-5p was analyzed using qRT-PCR assay in PC tissues and their corresponding adjacent samples $\left(\mathrm{n}=139,{ }^{* * *} \mathrm{P}<0.001\right)$. (B) miR-34a-5p expression was measured by $\mathrm{qRT}$-PCR assay in different tumor-node-metastasis (TNM) stages $\left({ }^{*} \mathrm{P}<0.05,{ }^{* *} \mathrm{P}<0.01,{ }^{*} * * \mathrm{P}<0.001\right)$. (C) Patients with low miR-34a-5p expression had a worse prognosis than the patients with high miR-34a-5p expression $(\mathrm{P}<0.01)$. (D) miR-34a-5p expression was measured by qRT-PCR assay in 67 patients with $\mathrm{PC}$ in pre-operation and post-operation $(\mathrm{P}<0.001)$. $\mathrm{PC}$, pancreatic cancer.

Table III. Correlation between miR-34a-5p expression level $(\Delta \mathrm{Ct})$ and clinicopathological characteristics of the PC patients.

\begin{tabular}{lccc}
\hline & & miR-34a-5p & \\
Characteristics & $\mathrm{n}(\%)$ & Mean \pm SD & P-value \\
\hline Total no. of patients & 139 & & \\
Age (years) & & & 0.785 \\
$>60$ & $59(42.4)$ & $11.48 \pm 1.75$ & \\
$\leq 60$ & $80(57.6)$ & $11.39 \pm 2.03$ & \\
Sex & & & 0.202 \\
Male & $89(64.0)$ & $11.45 \pm 1.79$ & \\
Female & $50(36.0)$ & $11.04 \pm 1.84$ & \\
Lymphatic metastasis & & & $\mathbf{0 . 0 0 6}^{\mathrm{a}}$ \\
N0 & $94(67.6)$ & $11.06 \pm 1.64$ & \\
N1-N2 & $45(32.4)$ & $11.91 \pm 2.52$ & \\
Distal metastasis & & & 0.089 \\
M0 & $105(75.5)$ & $11.24 \pm 1.93$ & \\
M1 & $34(24.5)$ & $11.94 \pm 2.46$ & \\
TNM stage & & & $\mathbf{0 . 0 0 9}^{\mathrm{a}}$ \\
0 \& I \& II & $91(65.5)$ & $11.58 \pm 1.74$ & \\
III \& IV & $48(34.5)$ & $12.35 \pm 1.39$ & \\
\hline
\end{tabular}

${ }^{\mathrm{a}} \mathrm{P}<0.01$. TNM, tumor-node-metastasis. $\mathrm{PC}$, pancreatic cancer.
miR-34a-5p inhibitors blocked this decrease mediated by XIST (Fig. 6D). Meanwhile, we also found that overexpression of XIST accelerated cell migration and invasion, while miR-34a-5p mimics inhibited this acceleration induced by XIST; knockdown of XIST decreased cell migration and invasion, and miR-34a-5p inhibitors blocked this decrease mediated by XIST $(\mathrm{P}<0.001$; Fig. $6 \mathrm{E})$.

\section{Discussion}

Pancreatic cancer (PC) has been the main cause of cancerrelated death worldwide for several decades $(37,38)$. Mortality of PC is projected to surpass breast and colorectal cancer by 2030 in the US $(39,40)$. The prognosis for patients with PC is poor with a reduced 5-year overall survival, and the median survival of patients with untreated PC is only 6 months with an extremely low percentage of long-term-surviving patients (41-43). However, at present, effective therapeutic strategies for patients with PC have been difficult to identify.

Emerging studies have identified numerous genes which are involved in the pathogenesis of human PC, and lncRNAs are important $(12,14,44-46)$. Numerous studies have indicated that IncRNAs participate in the biological processes of various cancer cells, including cell proliferation, development, apoptosis and metastases (47-49). For example, IRAIN was found to promote proliferation and suppress the apoptosis of PC cells (48); MALAT-1 accelerates cell growth, migration and invasion in PC (47). Nevertheless, the mechanisms of long non-coding RNA XIST in PC are not clear. 

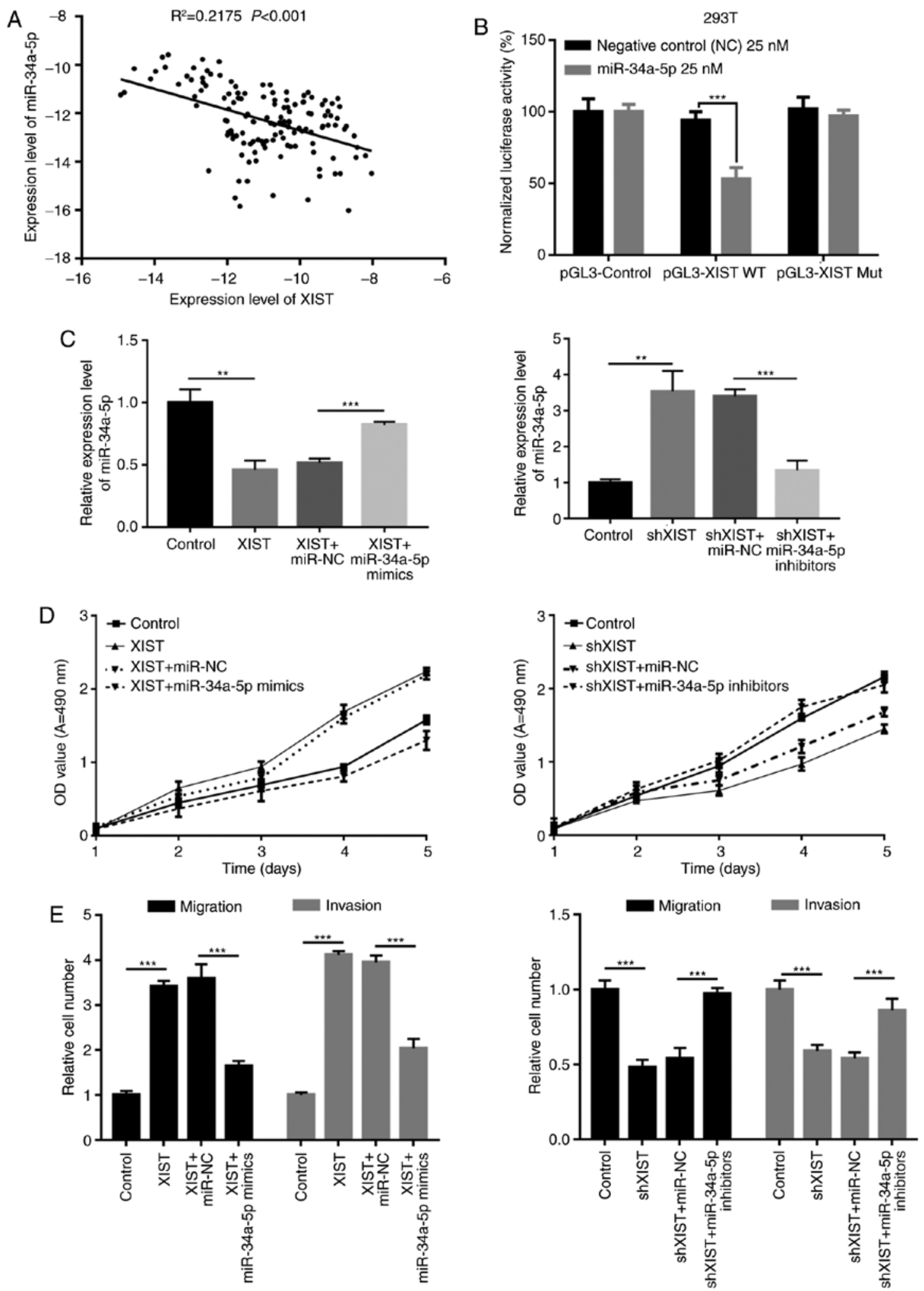

Figure 6. miR-34a-5p abrogates the facilitation of malignant behavior mediated by XIST. (A) The correlation between miR-34a-5p and XIST was determined by Pearson's correlation coefficient in clinical samples $\left(\mathrm{R}^{2}=0.2175, \mathrm{P}<0.001\right)$. (B) Luciferase reporter assay was performed in $293 \mathrm{~T}$ cells co-transfected with luciferase construct (pGL3-Control, pGL3-XIST WT or pGL3-XIST-Mut) and negative control (miR-control) or miR-34a-5p mimics, respectively $\left({ }^{* * * *} \mathrm{P}<0.001\right)$. (C) BxPC-3 cells were transfected with control, XIST, XIST plus miR-NC or XIST plus miR-34a-5p mimics; PANC-1 cells were transfected with control, shXIST, shXIST plus miR-NC or shXIST plus miR-34a-5p inhibitors, respectively. qRT-PCR assay was used to detect miR-34a-5p expression. GAPDH was used as control $\left({ }^{* *} \mathrm{P}<0.01,{ }^{* * *} \mathrm{P}<0.001\right)$. (D) MTT assay was performed to determine the viability of BxPC-3 and PANC-1 cells treated as in C. (E) The migration and invasion abilities were detected by Transwell assays in BxPC-3 and PANC-1 cells treated as in $\mathrm{C}\left({ }^{* * *} \mathrm{P}<0.001\right)$. XIST, $\mathrm{X}$ inactive-specific transcript;

MicroRNAs (miRNAs) have been demonstrated to be involved in the pathogenesis of many diseases, including cancers $(50)$, infections $(51,52)$ and diabetes $(53)$. In addi- tion, the differences in miRNA expression have regulatory functions in post-transcriptional modification or degradation of their target genes by binding to complementary regions 
in the 3'-untranslated region (UTR) of their target mRNA transcripts $(54,55)$. Previous studies have demonstrated that miRNAs play a crucial role in pancreatic development and function. For example, miR-34a-5p inhibits colorectal cancer metastasis and is related to patient recurrence (56); miR-34a-5p enhances the multi-drug resistance of osteosarcoma (57); miR-34a-5p promotes chemoresistance of osteosarcoma (58).

In the present study, we found that XIST was significantly upregulated in human PC tissues and PC cell lines, compared with that noted in the adjacent normal tissues and $\mathrm{HBE}$ normal lung epithelial cell line. Our result also indicated that XIST expression was markedly higher at later stages of tumor development and in pre-operation patients with PC. In addition, XIST significantly increased PC cell viability, G1-G0 phase arrest,cell proliferation, migration and invasion and inhibited cell apoptosis in vitro, while XIST knockdown had opposite effects. The in vivo studies demonstrated that XIST downregulation suppressed tumor growth. All of these data indicate that XIST plays an important role in the development of PC. However, the underlying mechanism by which XIST mediates gene expression and participates in tumorigenesis remains to be clarified. Recently, the ceRNA hypothesis proposed that lncRNAs communicate with other protein-coding RNA transcripts via shared common miRNA binding sites (32). According to the ceRNA hypothesis, miRNA complementary base pairing with XIST was predicted by starBase and TargetScan, and miR-34a-5p was identified. Quantitative real-time PCR showed that the expression of miR-34a-5p in BxPC-3 cells was decreased upon overexpresion of XIST, however increased by transfected with miR-34a-5p mimic. In PANC-1 cells, the expression of miR-34a-5p was increased upon knockdown of XIST; however the expression of miR-34a-5p was suppressed by miR-34a-5p inhibitor. Therefore, we suggested that XIST is a direct target of miR-34a-5p and there was interactive suppression between them. Furthermore, we used Transwell chamber inserts to explore the effects of miR-34a-5p and XIST on the migration and invasion of BxPC-3 and PANC-1 cells. The results showed that the transfection of the miR-34a-5p mimic abrogated the XIST-promoted BxPC-3 cell migration and invasion. However, transfection of the miR-34a-5p inhibitor hampered shXISTdecreased PANC-1 cell migration and invasion.

lncRNA XIST functions a tumor-promoting gene in PC. It promotes cell proliferation and invasion in $\mathrm{PC}$ by directly targeting and suppressing tumor-suppressor miR-34a-5p. Consequently, XIST could be a potential target for the prevention of the metastasis of PC.

\section{Competing interests}

The authors declare that they have no competing interests.

\section{References}

1. Ryan DP, Hong TS and Bardeesy N: Pancreatic adenocarcinoma. N Engl J Med 371: 1039-1049, 2014.

2. Egawa S, Toma H, Ohigashi H, Okusaka T, Nakao A, Hatori T, Maguchi H, Yanagisawa A and Tanaka M: Japan pancreatic cancer registry; 30th year anniversary: Japan pancreas society. Pancreas 41: 985-992, 2012.

3. Luo J, Xiao L, Wu C, Zheng Y and Zhao N: The incidence and survival rate of population-based pancreatic cancer patients: Shanghai Cancer Registry 2004-2009. PLoS One 8: e76052, 2013.
4. Boon RA, Jae N, Holdt L and Dimmeler S: Long non-coding RNAs: From clinical genetics to therapeutic targets? J Am Coll Cardiol 67: 1214-1226, 2016.

5. Qiu MT, Hu JW, Yin R and Xu L: Long non-coding RNA: An emerging paradigm of cancer research. Tumour Biol 34: 613-620, 2013.

6. Spizzo R, Almeida MI, Colombatti A and Calin GA: Long non-coding RNAs and cancer: A new frontier of translational research? Oncogene 31: 4577-4587, 2012.

7. Huang X, Zhi X, Gao Y, Ta N, Jiang H and Zheng J: LncRNAs in pancreatic cancer. Oncotarget 7: 57379-57390, 2016.

8. Peng W, Gao W and Feng J: Long non-coding RNA HULC is a novel biomarker of poor prognosis in patients with pancreatic cancer. Med Oncol 31: 346, 2014

9. Zhan HX, Wang Y, Li C, Xu JW, Zhou B, Zhu JK, Han HF, Wang L, Wang YS and Hu SY: LincRNA-ROR promotes invasion, metastasis and tumor growth in pancreatic cancer through activating ZEB1 pathway. Cancer Lett 374: 261-271, 2016.

10. Cheng Y, Jutooru I, Chadalapaka G, Corton JC and Safe S: The long non-coding RNA HOTTIP enhances pancreatic cancer cell proliferation, survival and migration. Oncotarget 6: 10840-10852, 2015.

11. Kim K, Jutooru I, Chadalapaka G, Johnson G, Frank J, Burghardt R, Kim S and Safe S: HOTAIR is a negative prognostic factor and exhibits pro-oncogenic activity in pancreatic cancer. Oncogene 32: 1616-1625, 2013.

12. Liu JH, Chen G, Dang YW, Li CJ and Luo DZ: Expression and prognostic significance of lncRNA MALAT1 in pancreatic cancer tissues. Asian Pac J Cancer Prev 15: 2971-2977, 2014.

13. Ma C, Nong K, Zhu H, Wang W, Huang X, Yuan Z and Ai K: H19 promotes pancreatic cancer metastasis by derepressing let-7's suppression on its target HMGA2-mediated EMT. Tumour Biol 35: 9163-9169, 2014.

14. Qu S, Yang X, Song W, Sun W, Li X, Wang J, Zhong Y, Shang R, Ruan B, Zhang Z, et al: Downregulation of lncRNA-ATB correlates with clinical progression and unfavorable prognosis in pancreatic cancer. Tumour Biol 37: 3933-3938, 2016.

15. Smola MJ, Christy TW, Inoue K, Nicholson CO, Friedersdorf M, Keene JD, Lee DM, Calabrese JM and Weeks KM: SHAPE reveals transcript-wide interactions, complex structural domains, and protein interactions across the Xist lncRNA in living cells. Proc Natl Acad Sci USA 113: 10322-10327, 2016.

16. Tantai J, Hu D, Yang Y and Geng J: Combined identification of long non-coding RNA XIST and HIF1A-AS1 in serum as an effective screening for non-small cell lung cancer. Int J Clin Exp Pathol 8: 7887-7895, 2015.

17. Yao Y, Ma J, Xue Y, Wang P, Li Z, Liu J, Chen L, Xi Z, Teng H, Wang Z, et al: Knockdown of long non-coding RNA XIST exerts tumor-suppressive functions in human glioblastoma stem cells by up-regulating miR-152. Cancer Lett 359: 75-86, 2015.

18. Chen DL, Ju HQ, Lu YX, Chen LZ, Zeng ZL, Zhang DS, Luo HY, Wang F, Qiu MZ, Wang DS, et al: Long non-coding RNA XIST regulates gastric cancer progression by acting as a molecular sponge of miR-101 to modulate EZH2 expression. J Exp Clin Cancer Res 35: 142, 2016.

19. Fang J, Sun CC and Gong C: Long non-coding RNA XIST acts as an oncogene in non-small cell lung cancer by epigenetically repressing KLF2 expression. Biochem Biophys Res Commun 478: 811-817, 2016.

20. Zhuang LK, Yang YT, Ma X, Han B, Wang ZS, Zhao QY, Wu LQ and Qu ZQ: MicroRNA-92b promotes hepatocellular carcinoma progression by targeting Smad7 and is mediated by long noncoding RNA XIST. Cell Death Dis 7: e2203, 2016.

21. Hayes EL and Lewis-Wambi JS: Mechanisms of endocrine resistance in breast cancer: An overview of the proposed roles of non-coding RNA. Breast Cancer Res 17: 40, 2015.

22. Garzon R, Calin GA and Croce CM: MicroRNAs in cancer. Ann Rev Med 60: 167-179, 2009.

23. Muluhngwi $\mathrm{P}$ and Klinge CM: Roles for miRNAs in endocrine resistance in breast cancer. Endocr Relat Cancer 22: R279-R300, 2015.

24. Filipska M, Skrzypski M, Bigda JJ and Jassem J: Biological role of prognostic microRNAs (miRNAs) in squamous lung cancer cell lines. J Thorac Oncol 10: S391-S391, 2015

25. Kara M, Yumrutas O, Ozcan O, Celik OI, Bozgeyik E, Bozgeyik I and Tasdemir S: Differential expressions of cancer-associated genes and their regulatory miRNAs in colorectal carcinoma. Gene 567: 81-86, 2015.

26. Zhang P, Zuo Z, Wu A, Shang W, Bi R, Jin Q, Wu J and Jiang L: miR-600 inhibits cell proliferation, migration and invasion by targeting p53 in mutant p53-expressing human colorectal cancer cell lines. Oncol Lett 13: 1789-1796, 2017. 
27. Ergun S and Oztuzcu S: Oncocers: ceRNA-mediated cross-talk by sponging miRNAs in oncogenic pathways. Tumour Biol 36: 3129-3136, 2015

28. Su X, Xing J, Wang Z, Chen L, Cui M and Jiang B: microRNAs and ceRNAs: RNA networks in pathogenesis of cancer. Chin J Cancer Res 25: 235-239, 2013.

29. Guo LL, Song CH, Wang P, Dai LP, Zhang JY and Wang KJ: Competing endogenous RNA networks and gastric cancer. World J Gastroenterol 21: 11680-11687, 2015.

30. Liang WC, Fu WM, Wong CW, Wang Y, Wang WM, Hu GX, Zhang L, Xiao LJ, Wan DC, Zhang JF and Waye MM: The lncRNA H19 promotes epithelial to mesenchymal transition by functioning as miRNA sponges in colorectal cancer. Oncotarget 6: 22513-22525, 2015.

31. Qi X, Zhang DH, Wu N, Xiao JH, Wang X and Ma W: ceRNA in cancer: Possible functions and clinical implications. J Med Genet 52: 710-718, 2015.

32. Xia T, Liao Q, Jiang X, Shao Y, Xiao B, Xi Y and Guo J: Long non-coding RNA associated-competing endogenous RNAs in gastric cancer. Sci Rep 4: 6088, 2014.

33. Jiao C, Song Z, Chen J, Zhong J, Cai W, Tian S, Chen S, Yi Y and Xiao Y: LncRNA-UCA1 enhances cell proliferation through functioning as a ceRNA of Sox4 in esophageal cancer. Oncol Rep 36: 2960-2966, 2016

34. Liu XH, Sun M, Nie FQ, Ge YB, Zhang EB, Yin DD, Kong R, Xia R, Lu KH, Li JH, et al: Lnc RNA HOTAIR functions as a competing endogenous RNA to regulate HER 2 expression by sponging miR-331-3p in gastric cancer. Mol Cancer 13: 92, 2014

35. Xia T, Chen S, Jiang Z, Shao Y, Jiang X, Li P, Xiao B and Guo J: Long non-coding RNA FERIL4 suppresses cancer cell growth by acting as a competing endogenous RNA and regulating PTEN expression. Sci Rep 5: 13445, 2015.

36. Livak KJ and Schmittgen TD: Analysis of relative gene expression data using real-time quantitative PCR and the $2^{-\Delta \Delta C \mathrm{~T}}$ method. Methods 25: 402-408, 2001.

37. Alzheimer's Association: 2013 Alzheimer's disease facts and figures. Alzheimers Dement 9: 208-245, 2013.

38. Malvezzi M, Bertuccio P, Levi F, La Vecchia C and Negri E: European cancer mortality predictions for the year 2013. Ann Oncol 24: 792-800, 2013.

39. Rahib L, Smith BD, Aizenberg R, Rosenzweig AB, Fleshman JM and Matrisian LM: Projecting cancer incidence and deaths to 2030: The unexpected burden of thyroid, liver, and pancreas cancers in the United States. Cancer Res 74: 2913-2921, 2014.

40. No authors listed: Cancer statistics JAMA 310: 982, 2013.

41. Siegel R, Ma J, Zou Z and Jemal A: Cancer statistics, 2014. CA Cancer J Clin 64: 9-29, 2014.

42. Hidalgo M: Pancreatic cancer. N Engl J Med 362: 1605-1617, 2010.

43. Vincent A, Herman J, Schulick R, Hruban RH and Goggins M: Pancreatic cancer. Lancet 378: 607-620, 2011.

44. Li X, Deng SJ, Zhu S, Jin Y, Cui SP, Chen JY, Xiang C, Li QY, He C, Zhao SF, et al: Hypoxia-induced lncRNA-NUTF2P3-001 contributes to tumorigenesis of pancreatic cancer by derepressing the miR-3923/KRAS pathway. Oncotarget 7: 6000-6014, 2016.
45. Müller S, Raulefs S, Bruns P, Afonso-Grunz F, Plötner A, Thermann R, Jäger C, Schlitter AM, Kong B, Regel I, et al: Next-generation sequencing reveals novel differentially regulated mRNAs, IncRNAs, miRNAs, sdRNAs and a piRNA in pancreatic cancer. Mol Cancer 14: 94, 2015.

46. Ye S, Yang L, Zhao X, Song W, Wang W and Zheng S: Bioinformatics method to predict two regulation mechanism: TF-miRNA-mRNA and lncRNA-miRNA-mRNA in pancreatic cancer. Cell Biochem Biophys 70: 1849-1858, 2014.

47. Jiao F, Hu H, Yuan C, Wang L, Jiang W, Jin Z, Guo Z and Wang L: Elevated expression level of long non-coding RNA MALAT-1 facilitates cell growth, migration and invasion in pancreatic cancer. Oncol Rep 32: 2485-2492, 2014.

48. Lian Y, Wang J, Feng J, Ding J, Ma Z, Li J, Peng P, De W and Wang K: Long non-coding RNA IRAIN suppresses apoptosis and promotes proliferation by binding to LSD1 and EZH2 in pancreatic cancer. Tumour Biol 37: 14929-14937, 2016.

49. Zheng S, Chen H, Wang Y, Gao W, Fu Z, Zhou Q, Jiang Y, Lin Q, Tan L, Ye H, et al: Long non-coding RNA LOC389641 promotes progression of pancreatic ductal adenocarcinoma and increases cell invasion by regulating E-cadherin in a TNFRSF10A-related manner. Cancer Lett 371: 354-365, 2016.

50. Hwang HW and Mendell JT: MicroRNAs in cell proliferation, cell death, and tumorigenesis. Br J Cancer 94: 776-780, 2006

51. Sullivan CS and Ganem D: MicroRNAs and viral infection. Mol Cell 20: 3-7, 2005

52. Staedel C and Darfeuille F: MicroRNAs and bacterial infection. Cell Microbiol 15: 1496-1507, 2013.

53. Kato M, Castro NE and Natarajan R: MicroRNAs: Potential mediators and biomarkers of diabetic complications. Free Radic Biol Med 64: 85-94, 2013.

54. He L and Hannon GJ: MicroRNAs: Small RNAs with a big role in gene regulation. Nat Rev Genet 5: 522-531, 2004.

55. Filipowicz W: RNAi: The nuts and bolts of the RISC machine. Cell 122: 17-20, 2005.

56. Gao J, Li N, Dong Y, Li S, Xu L, Li X, Li Y, Li Z, Ng SS, Sung JJ, et al: $\mathrm{miR}-34 \mathrm{a}-5 \mathrm{p}$ suppresses colorectal cancer metastasis and predicts recurrence in patients with stage II/III colorectal cancer. Oncogene 34: 4142-4152, 2015.

57. Pu Y, Zhao F, Wang H, Cai W, Gao J, Li Y and Cai S: MiR-34a-5p promotes the multi-drug resistance of osteosarcoma by targeting the CD117 gene. Oncotarget 7: 28420-28434, 2016.

58. Pu Y, Zhao F, Li Y, Cui M, Wang H, Meng X and Cai S: The miR$34 a-5 p$ promotes the multi-chemoresistance of osteosarcoma via repression of the AGTR1 gene. BMC Cancer 17: 45, 2017.

This work is licensed under a Creative Commons Attribution-NonCommercial-NoDerivatives 4.0 International (CC BY-NC-ND 4.0) License. 